\title{
Mapping genome-wide transcription factor binding sites in frozen tissues
}

\author{
Daniel Savic, Jason Gertz, Preti Jain, Gregory M Cooper and Richard M Myers*
}

\begin{abstract}
Background: Genome-wide maps of transcription factor binding sites in primary tissues can expand our understanding of genome function, transcriptional regulation, and genetic alterations that contribute to disease risk. However, almost all genome-wide studies of transcription factors have been in cell lines, and performing these experiments in tissues has been technically challenging and limited in throughput.

Results: Here we outline a simple strategy for mapping transcription factor binding sites in frozen tissues that utilizes dry pulverization of samples and is scalable for high-throughput analyses. We show that the method leads to accurate and reproducible chromatin immunoprecipitation next-generation sequencing (ChIP-seq) data, and is highly sensitive, identifying high-quality transcription factor binding sites from chromatin corresponding to only $5 \mathrm{mg}$ of liver tissue.
\end{abstract}

Conclusions: The enhanced reproducibility, robustness, and sensitivity of the dry pulverization method, in addition to the ease of implementation and scalability, makes ChIP-seq in primary tissues a widely accessible assay.

Keywords: ChIP-seq, Frozen tissues, Gene regulation, Genomics, Tissue specificity

\section{Background}

The assembly of a reference human genome coupled to the development of novel array and next-generation sequencing technologies has ushered in an era of highresolution approaches for mapping functional genomic features [1-4], and higher-order nuclear architecture $[5,6]$. In particular, cataloging genome-wide binding profiles of diverse DNA-binding proteins through nextgeneration sequencing of chromatin immunoprecipitated DNA, or chromatin immunoprecipitation next-generation sequencing (ChIP-seq) [7,8], has deepened our understanding of transcription factor involvement in gene regulation. Although cell lines have played a seminal role in describing how transcription factors interact with the genome [1,2,9-13], transcription factor characterization in primary tissues is a critical next step. While cell models allow for a focused and controlled biological system that can be manipulated in a variety of ways, the use of cancerderived or transformed cells [14], as well as artificial culture conditions, can make direct biological inferences about normal in vivo states challenging. Despite being

\footnotetext{
* Correspondence: rmyers@hudsonalpha.org
HudsonAlpha Institute for Biotechnology, 601

HudsonAlpha Institute for Biotechnology, 601 Genome Way, Huntsville, AL 35806, USA
}

more complex and heterogeneous, tissues are derived from an in vivo context that is subject to physiological conditions, and therefore the direct analysis of primary tissues should provide more relevant insights into endogenous biological functions. Indeed, several influential ChIP-seq studies have been successfully performed in diverse mouse tissues [15-18], as well as normal [19] and diseased [20] human tissue samples. These investigations have illustrated the power of genomic assays in primary tissues for characterizing basic cellular functions and the genomic hallmarks common to disease states.

However, a major barrier to the broader utilization of ChIP-seq in tissues is that previously published strategies are much more technically challenging and labor intensive than similar protocols in cell lines. These tissue approaches have primarily relied on a mincing technique, wherein adult tissues are diced with a razor prior to fixation and homogenized in a Dounce homogenizer after fixation [21,22]. This dicing method is technically difficult and time consuming, making it less tractable for large-scale projects. The mincing approach can also lead to substantial sample loss through excessive handling of the tissue, while cross-contamination concerns diminish throughput. Importantly, differences in minced tissue 
sizes within and across samples can lead to heterogeneous fixation, which could limit reproducibility, assay sensitivity and overall data quality. This aspect may be particularly problematic for rare or scarce tissue samples. To circumvent the outstanding challenges with available methods, here we outline a ChIP-seq protocol for frozen tissue analyses that is simple, efficient, and involves minimal effort, making it easily implementable and amenable to higher throughput. We find the method to be robust across diverse tissue types and highly reproducible for both a general transcription factor (Rnap2) and sequence-specific factors (Ctcf and Rxro). We also determined that our strategy is sensitive, accurately and reproducibly identifying binding sites with chromatin amounts that corresponded to only 5 mg of mouse liver ( $\approx 675,000$ hepatocytes), far lower than previous protocols demand for liver tissue [21]. The approach also captures tissue specificity while further recapitulating results from previous genomic analyses.

\section{Results}

\section{ChIP-seq in mouse tissues}

To perform ChIP-seq in frozen tissues while minimizing tissue handling and cross-contamination concerns, we developed an approach that uses dry pulverization (Figure 1 and Methods). Tissues are placed in bags that can withstand high force and low temperatures and are pulverized on dry ice while repeatedly being cooled with liquid nitrogen. The repeated freezing with liquid nitrogen ensures that the tissue remains both frozen and brittle, facilitating the pulverization process. The resulting tissue powder is fixed with formaldehyde at room temperature and subsequent ChIP experimentation is performed in a manner identical to that in cell lines [23] (see Methods). Overall, the dry pulverization frozen tissue ChIP-seq protocol is simple and requires minimal steps, time and effort, making it easily implementable and scalable to higher throughput analyses. Supporting the enhanced throughput of the method, all murine samples were fixed in large batches that consisted of several distinct tissue sample types, and took approximately one hour to pulverize and fix.

To evaluate the dry pulverization technique, we tested mouse liver tissue with antibodies that target RNA polymerase II (Rnap2), the insulator CCCTC-binding factor (Ctcf) and the Retinoid X receptor $\alpha$ (Rxr $\alpha)$ nuclear receptor. We chose these factors to determine the feasibility of our strategy in defining genome-wide maps of distinct classes of DNA-binding proteins that are widely expressed in different tissue types, including RNA polymerases, canonical transcription factors, and proteins involved in maintaining genome structure.

Using a common set of parameters for pulverization, fixation, and ChIP for all experiments (see Methods), we identified thousands of binding sites for Rnap2, Ctcf, and Rxro in mouse liver (Figure 2A and Additional file 1: Table S1). For the sequence-specific factors (Ctcf and Rxro), we determined that binding sites were enriched for their known canonical binding motifs (Figure 2B). We also performed ChIP analyses with independent biological replicates for each protein and found that more than $87 \%$ of transcription factor binding site overlap between replicates, illustrating the high reproducibility of the method (Figure 2C and Additional file 1: Table S1). As an additional validation of the technique, we performed ChIP-seq to identify CCAAT/enhancer-binding protein $\alpha(\operatorname{Cebp} \alpha)$ transcription factor binding sites, because this protein has an integral role in hepatic energy metabolism [24], and its binding pattern has been previously mapped in mouse livers [25], allowing for a direct comparison. Consistently with the results obtained from the other factors, we identified highly reproducible 


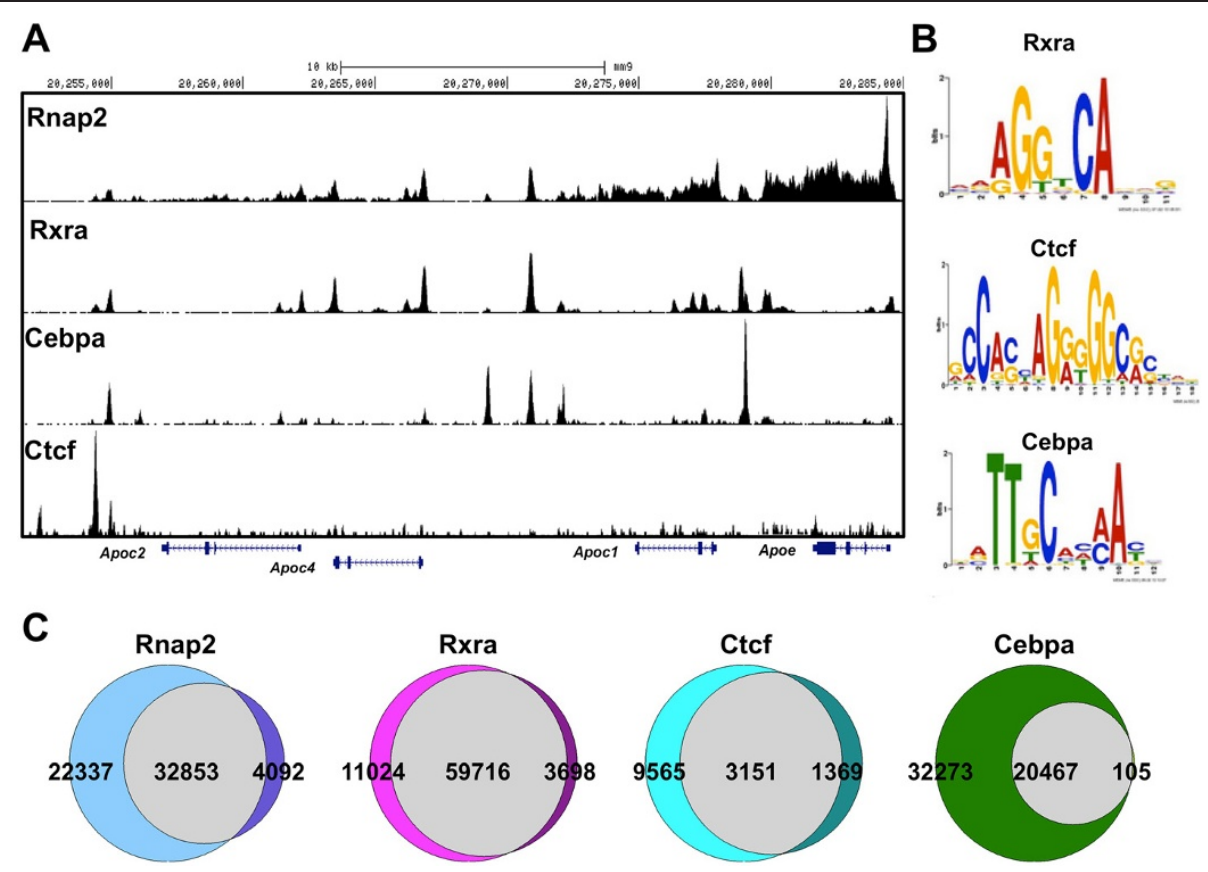

Figure 2 ChIP-seq in murine liver. (A) ChIP raw sequencing read enrichments for RNA polymerase II (Rnap2), Retinoid X receptor a (Rxra), CCAAT/enhancer-binding protein a (Cebpa), and the CCCTC-binding factor (Ctcf) at an apolipoprotein cluster on mouse chromosome 7. (B) Images of the canonical motifs identified by multiple expectation maximization for motif elicitation (MEME) in liver. (C) Venn diagrams illustrate the degree of shared binding sites between liver biological replicates.

Cebpa binding sites enriched for the canonical motif (Figure 2B and Additional file 1: Table S1). We also evaluated the quality of our sequencing libraries by calculating library complexities, or the fraction of aligned sequences that map to a unique genome location compared with a randomly selected set of 10 million aligned sequences. For all ChIP experiments, including replicates, we found that our sequencing libraries harbored a high degree of complexity (Additional file 1: Table S1).

Given the success of the dry pulverization ChIP-seq protocol in liver, we assessed the performance of the assay in mouse brain, small intestine, and skeletal muscle samples with Rnap2, Ctcf, and Rxra antibodies. The dry pulverization approach, with identical parameters for pulverization, fixation and ChIP, produced high-quality ChIP-seq data for each factor across all tissues despite their distinct histology (Additional file 1: Table S1), highlighting the robustness of the technique. For sequence-specific factors, the sites we identified were enriched for their canonical binding motifs (Additional file 2: Figure S1). Like our observations in liver, our results on binding sites were highly reproducible, with more than $75 \%$ of binding sites being shared between all pairs of biological replicates (Additional file 1: Table S1). We also found a high sequence complexity for all sequencing libraries (Additional file 1: Table S1). Collectively, these data indicate that the dry pulverization
ChIP-seq method is robust across distinct tissue types and can reproducibly identify binding sites for distinct classes of DNA-binding proteins.

\section{Tissue specificity of genome-wide binding sites}

To assess whether the dry pulverization approach can capture meaningful biological information, we assessed the functional relevance of the binding data through intratissue and intertissue analyses. We evaluated the extent of binding colocalization between the three predominant DNA-binding proteins that we tested (Rnap2, Ctcf, and Rxro) within each tissue (Figure 3A). We found that a higher proportion of Rxra binding sites are shared with Rnap2 than with Ctcf in all tissues examined ( $44.8 \%$ vs. $6.7 \%$ in liver, $42.5 \%$ vs. $14.1 \%$ in brain, $72.5 \%$ vs. $32.9 \%$ in small intestine, $48.5 \%$ vs. $1.4 \%$ in skeletal muscle) (Figure 3A). These data are consistent with the distinct role of Ctcf in chromatin insulation and enhancer blocking [26], while the shared Rnap2/Rxra sites may reflect indirect association via binding of Rxr $\alpha$ to sites near expressed genes and direct associations involving the recruitment of Rnap2 to promoter-distal Rxr $\alpha$ bound enhancer sequences [27,28].

We next performed cross-tissue analyses by determining the proportion of Rnap2, Ctcf and Rxr $\alpha$ binding sites that were shared between tissues (Figure $3 \mathrm{~B}$ and $3 \mathrm{C}$ ). Rnap2 and Rxra binding sites exhibited strong tissue 

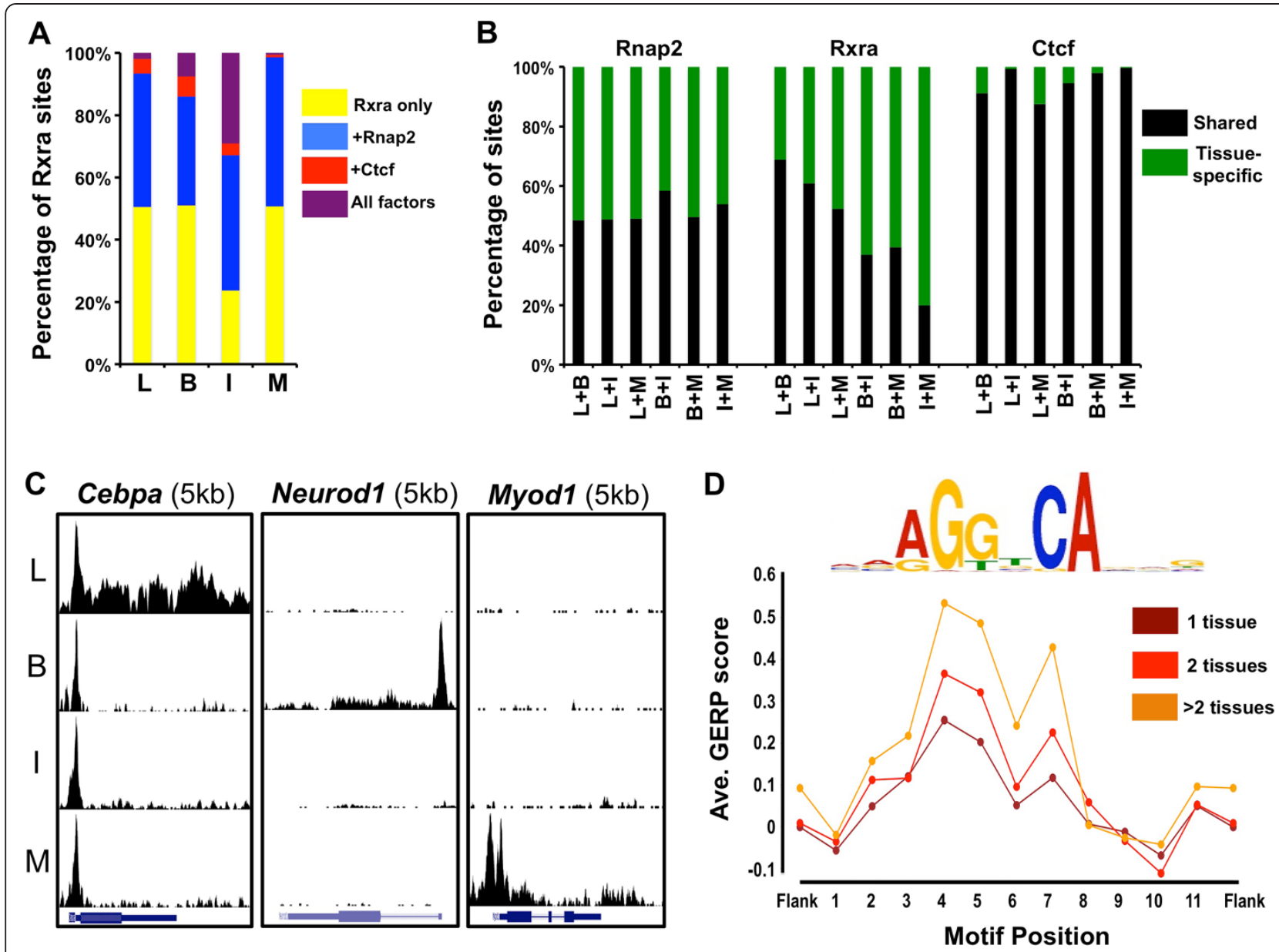

Figure 3 ChIP-seq analyses across diverse murine tissues. (A) Percentage of Rxra binding sites shared with Rnap2 (blue), Ctcf (red) or both Rnap2 and Ctcf (purple) in liver (L), brain (B), small intestine (I) and skeletal muscle (M). Rxra binding sites that do not colocalize with Rnap2 and Ctcf are shown in yellow. (B) Analysis of shared binding sites for Rnap2, Rxra, and Ctcf between all pairwise tissue comparisons. The two tissues utilized for each comparison are given on the $x$ axis. Shared binding sites are shown in black while tissue-specific sites are in green. (C) ChIP-seq raw sequencing read enrichments for Rnap2 at distinct genes illustrate tissue specificity of gene expression. Gene names and window sizes are given above. (D) Canonical motif genomic evolutionary rate profiling (GERP) scores at tissue-specific binding sites (dark red) and binding sites shared by two (red) or more (orange) tissues. The corresponding motif sequence is shown above the graph. GERP scores are significantly higher within bound Rxra motifs relative to positions that are not within a motif but are within $250 \mathrm{bp}$ of a binding site summit $\left(P<2.2 \times 10^{-16}\right.$, one-sided $t$ test); further, there is a highly significant correlation between GERP score and position-specific motif dependencies on a particular nucleotide, with less degenerate positions being more highly conserved $\left(P<2.2 \times 10^{-16}\right.$, simple linear regression between GERP scores and the maximum individual nucleotide score at each position in the Rxra motif position-specific weight matrix).

specificity, with between-tissue overlap rates ranging from $48.5 \%$ to $58.4 \%$ for Rnap2, and $20 \%$ to $68.9 \%$ for Rxro (Figure 3B); on average, half of the Rnap2 and Rxro sites are tissue-specific. In contrast to the tissue specificity of Rnap2 and Rxro, Ctcf binding was largely similar across tissues, with typical overlap rates exceeding $90 \%$ between tissue pairs (Figure 3B). In support of these findings, we also analyzed Rnap2 enrichment at a set of tissue-specific genes and determined that active transcription was limited to the appropriate tissue type (Figure 3C). These observations are consistent with previous studies showing stable Ctcf binding patterns across cell types [29].
We subsequently performed gene ontology analyses for the binding profiles of Rnap2 and Rxro. Consistently with the colocalization results that pointed to pronounced tissue specificity, genes near Rnap2 and Rxr $\alpha$ binding sites are enriched for distinct biological functions across tissues, and these pathways largely reflect known processes in each tissue (Additional file 2: Figures S2 and S3). For instance, Rnap2 binding sites are enriched for genes related to fatty acid metabolism in liver and muscle cell development in the muscle, while genes near Rxro binding sites in liver are enriched for lipid and cholesterol metabolic processes, and Rxra binding in skeletal muscles enriches for genes involved 
in actin filament-based processes and actin cytoskeleton organization.

We also examined sequence conservation at Rxro binding sites using genomic evolutionary rate profiling (GERP) scores [30]. In particular, we assessed the difference in conservation between canonical motifs at Rxro binding sites that are tissue-specific and sites that are bound by Rxra in two or more tissues (Figure 3D). The data show that, while canonical motifs at Rxro binding sites are more highly conserved than flanking sequences, the degree of conservation is particularly increased at sites bound in multiple tissues. These data suggest a stronger selective pressure on pleiotropic binding sites relative to tissue-specific sites. In line with the canonical motif data, a similar trend in conservation between tissue-specific and common binding sites is observed when GERP scores are tabulated relative to the binding site peak summit, whether or not a motif is present (Additional file 2: Figure S4).

To define the relationship between each factor across the four tissues that were examined, we looked at the pairwise Spearman rank correlations of normalized read depth within a collective list of binding sites (Figure 4). The resulting correlation matrix captures tissue specificity as the canonical transcription factors (Rxr $\alpha$ and Cebp $\alpha$ ) and Rnap 2 display a correlation primarily driven by tissue type, while the Ctcf binding patterns form an independent cluster, further supporting the limited cell-type specificity of Ctcf binding [29].
Replication of results from independent genomic datasets We used publicly available genomic datasets from mouse tissues, including ChIP-seq, DNaseI hypersensitivity, and RNA-seq to further evaluate how well our data replicate previous analyses. We obtained Rnap2 and Ctcf ChIPseq data from the Mouse ENCODE Consortium for liver, brain (by combining cortex and cerebellum datasets) and small intestine to assess the fraction of binding sites that are shared [17]. Importantly, these transcription factor binding site datasets were identified using the mincing strategy and therefore allow a direct comparison with the pulverization method. We determined that our ChIP-seq results and mouse ENCODE data exhibited high concordance, as 60 to $75 \%$ of Rnap 2 binding sites and 78 to $98 \%$ of Ctcf binding sites colocalized between datasets in the three tissues examined (Additional file 2: Figure S5). We also evaluated Cebp $\alpha$ binding in liver and determined that more than $90 \%$ of our liver Cebp $\alpha$ binding sites $(19,155$ out of 20,467$)$ were identified in an independent study [25].

Given the lack of available Rxro ChIP-seq data in murine tissues, we determined the proportion of Rxro binding sites that coincided with diverse epigenetic modifications identified by the Mouse ENCODE Consortium in liver, brain (by combining cortex and cerebellum data sets) and small intestine [17]. For this analysis, we used histone 3 lysine 4 mono-methylation (H3K4me1) marks, as this modification is found at active chromatin regions and is associated with regulatory elements [31,32], as well as

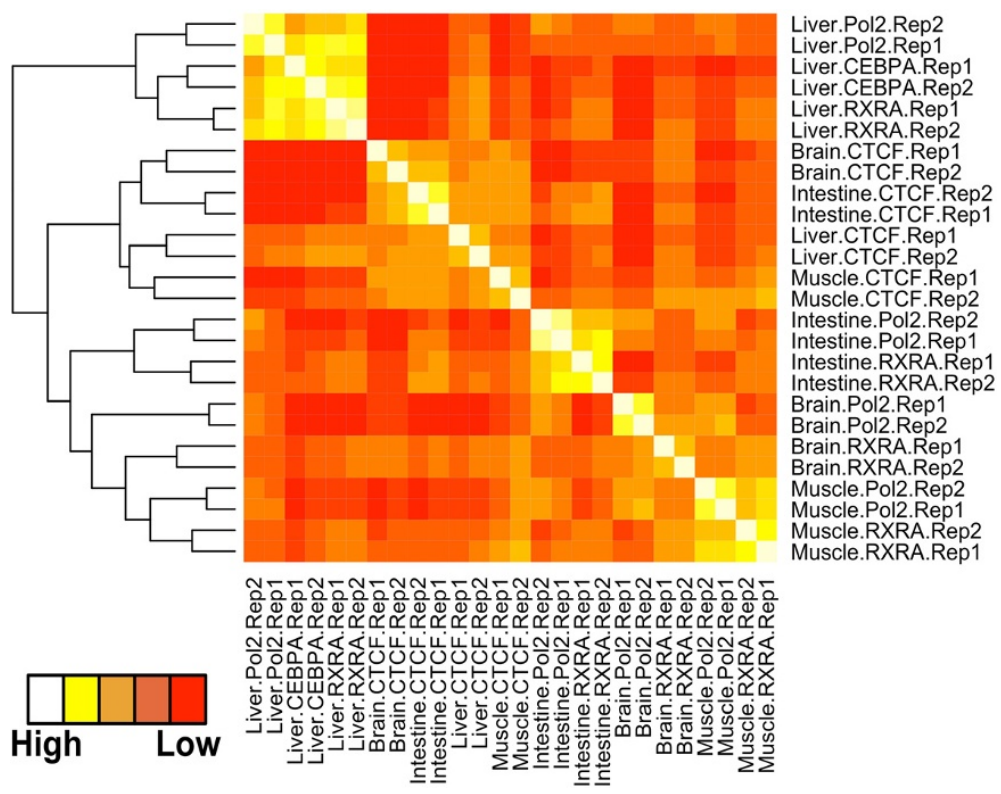

Figure 4 Correlation matrix between ChIP-seq experiments. Heat map displaying Spearman rank correlations between all pairwise comparisons for all tissues and ChIPs. Spearman correlations were calculated using the normalized read depth across the entire set of binding sites identified for all ChIP-seq experiments. RNA polymerase II, Pol2; CCCTC-binding factor, Ctcf; Retinoid X receptor a; Rxra; CCAAT/enhancer-binding protein a, CEBPa. 
histone 3 lysine 27 tri-methylation (H3K27me3) modifications that are involved in epigenetic silencing [33]. In line with the identification of putatively active cis-regulatory enhancer elements, Rxro binding sites consistently colocalized with active H3K4me1 marks $(84.1 \%$ in liver, $51.4 \%$ in brain, and $87.2 \%$ in small intestine), in contrast with H3K27me3 repressive modifications $(1.7 \%$ in liver, $1 \%$ in brain, and $1 \%$ in small intestine). Confirming the high ChIP-seq data quality, similar enrichments with histone modifications were also obtained using Rnap2 and Ctcf datasets (Additional file 2: Figure S6).

We also calculated the proportion of Rnap2, Ctcf, and Rxro binding sites that associated with regions of open chromatin as identified by DNaseI hypersensitivity $[34,35]$ from liver, brain, and skeletal muscle tissues performed by the Mouse ENCODE Consortium. The vast majority of Rnap2 (83.4\%), Ctcf (86.8\%), and Rxra (87.2\%) sites across these three tissues were situated in regions of open chromatin (Additional file 2: Figure S7). The degree of background or non-specific colocalization was also determined by performing cross-tissue comparisons with ChIP-seq and DNaseI hypersensitivity datasets. We found a significantly stronger enrichment of Rnap2 and Rxro binding sites with open chromatin regions from the same tissue type compared with cross-tissue comparisons $(P<0.05$, two-sided Student's $t$ test), while Ctcf binding enrichment with open chromatin was not significantly different between intratissue and intertissue comparisons, further confirming the limited tissue specificity of Ctcf [29].

We also used a publicly available RNA-seq dataset [36] to evaluate the association between Rnap2 ChIP-seq signal and the level of gene expression. For this analysis, we determined the normalized read depth for Rnap2 at gene promoters in liver, brain and skeletal muscle and correlated these values with gene expression measurements (reads per kilobase per million mapped reads or RPKMs) in the same tissue (Additional file 2: Figure S8). Both Rnap2 ChIP-seq replicates from all three tissues exhibited extensive rank correlation with gene RPKMs (0.78 in liver, 0.70 in brain, and 0.77 in muscle), suggesting that our Rnap2 ChIP-seq data at promoters is an accurate predictor of gene expression levels in the same tissue.

\section{Tissue ChIP-seq input requirements}

To measure the sensitivity of the dry pulverization ChIP method, we performed a titration of mouse liver tissue by performing ChIP-seq targeting Rxro. For the titration, we sonicated $100 \mathrm{mg}$ fixed liver powder and subsequently performed ChIP-seq on aliquots that corresponded to 50, 25, 10 and $5 \mathrm{mg}$ of tissue. Binding sites that were enriched for the canonical Rxra binding motif were identified across all tissue input amounts (Figure 5A). Based on previous estimates of hepatocyte number in the mouse liver [37], these data suggest that our assay can generate genome-wide binding profiles using chromatin from as little as $\approx 675,000$ hepatocytes. We determined that each tissue input maintained strong reproducibility, including $92 \%$ of binding sites that were shared even between $5 \mathrm{mg}$ biological replicates (Additional file 1: Table S1). Through pairwise comparisons between different tissue amounts, we found that, in each case, more than $90 \%$ of binding sites were concordant (Additional file 2: Figure S9). To validate these observations further, we looked at Spearman rank correlations of ChIP-seq signal across tissue amounts and consistently identified strong correlations (Figure 5B). As we anticipated, our data exhibited a positive correlation between the number of binding sites identified and the sample input amount, as increasing amounts of input chromatin lead to a higher number of binding sites (Figure $5 \mathrm{C}$ ). Despite the change in binding sites, we identified more than 25,000 reproducible Rxro binding sites with only a small fraction of chromatin that represented $5 \mathrm{mg}$ liver tissue. Remarkably, the assay also retained a high library complexity across all samples (Additional file 1: Table S1). These results suggest that the dry pulverization technique is highly robust and can maximize the number of ChIP-seq experiments performed on limited or rare amounts of frozen tissue samples, such as patient samples.

\section{Discussion}

While many ChIP-seq analyses have been performed in cell lines, primary tissues have not been extensively assayed, even though they may serve as a more appropriate model for evaluating biological function in an organismal context. The labor-intensive and technically challenging nature of previously described protocols involving tissues is likely to have limited the number of primary tissue ChIP-seq investigations. Here we have described and systematically assessed a dry pulverization method for efficiently performing ChIP-seq in frozen mammalian tissues. Importantly, similar pulverization ChIP-seq strategies have been successfully performed in Drosophila [38] and Caenorhabditis elegans [39] tissues.

We demonstrate that the pulverization strategy generates high-quality data and is highly reproducible, with more than $75 \%$ of binding sites being shared across all biological replicates. Our results are also concordant with transcription factor binding site ChIP-seq data obtained using the mincing strategy, as well as DNaseI hypersensitive sites, histone modifications, and gene expression profiles. However, the advantage of the dry pulverization method is in its simplicity, requiring minimal effort and time, thereby making it efficient and adaptable for largescale projects. Tissue samples can be pulverized in large batches and tissue powder can be stored upon pulverization, prior to crosslinking, allowing laboratories to perform these assays in a higher throughput manner. We 


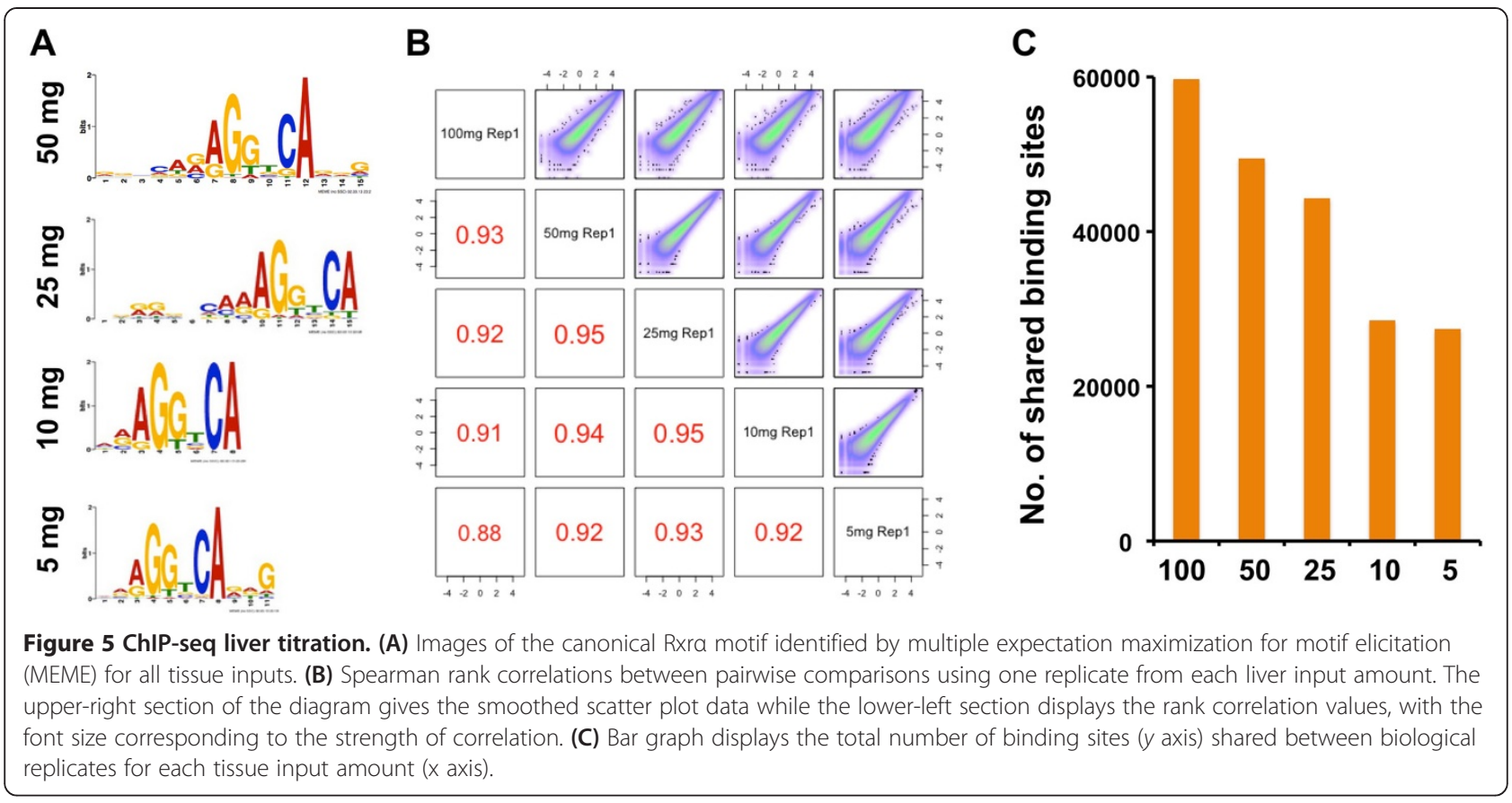

also performed all tissue preparations in large batches consisting of several distinct tissue types, and these took only 1 hour to perform. The approach also ensures that tissues remain frozen during the preparation prior to fixation, controlling for potential tissue degradation during sample preparation. The utilization of tissue powder rather than diced tissue for fixation ensures a more homogenous and reproducible fixation, limiting technical variance that may occur through differences in fixation efficiency between tissue replicates or across distinct tissue types. Supporting the advantages of the pulverization method, we determined that the assay was robust, as we successfully performed all ChIP-seq assays by relying on a common set of experimental conditions, despite the different histology and physical characteristics of the tissues examined (see Methods). Collectively, these data suggest that the dry pulverization method captures both meaningful and accurate biological information, yet involves fewer steps and less effort than standard protocols.

We also evaluated the sensitivity of dry pulverization by titrating mouse liver tissue. We demonstrate that the dry pulverization ChIP-seq method is highly sensitive and can identify binding sites from chromatin that corresponds to as little as $5 \mathrm{mg}$ liver tissue, or $\approx 675,000$ hepatocytes, substantially less than previous recommendations for liver tissue [21]. The uniform fixation that occurs through the use of tissue powder may be a contributing factor for the enhanced sensitivity of the pulverization method. In light of these observations, dry pulverization is therefore highly advantageous for handling scarce or limited tissues, such as clinical samples, as it allows for a large number of ChIP-seq experiments to be performed from one sample. However, the lower limit of input amount will probably fluctuate between distinct tissues, as this threshold will be dependent on the nuclear density of a given tissue, in addition to antibody quality. The parameters we used should serve as a starting point for subsequent investigations and additional optimization of conditions may be required for unique tissues not assessed in our study. Although the tissue samples we used for our analyses weighed at least $200 \mathrm{mg}$ prior to pulverization, obtaining powder from smaller initial tissue sample amounts with minimal tissue loss is feasible (see Methods), but downstream sonication and immunoprecipitation conditions may need additional optimization.

The technological advancements over the last decade have allowed for high-resolution studies of genomic processes and these large-scale analyses have led to a deeper understanding of the complexity involved in gene regulation [1,2,9-13]. However, the validation of phenomena observed in cells and the delineation of novel functions in more complex tissues is a critical step for accurately characterizing endogenous biological processes, and for defining genomic anomalies in diseased tissues. We believe that the dry pulverization tissue ChIP-seq approach outlined in this study can facilitate these important next steps.

\section{Conclusions}

We report a dry pulverization method for ChIP-seq analysis of frozen tissues that is robust, reproducible and requires minimal input. The simplicity of dry pulverization 
and the fact that this technique is amenable to higher throughput analyses collectively makes tissue ChIP-seq analyses widely feasible.

\section{Methods}

\section{Tissue pulverization and fixation}

Tissue samples from $\mathrm{C} 57 \mathrm{BL} / 6 \mathrm{~J}$ mice at 8 weeks of age were obtained from Jackson Laboratories. Mice were cervically dislocated and tissues were dissected and snap frozen in liquid nitrogen. Tissues were subsequently stored at $-80^{\circ} \mathrm{C}$ until pulverization. Prior to pulverization, a hammer and a metallic block were chilled on dry ice for 20 to 30 minutes. During this preparatory cooling, the metallic block was secured by packing the dry ice around the block to ensure that it would not move during pulverization. Frozen tissue samples $(\approx 200$ to $800 \mathrm{mg}$ each) were pulverized in Covaris tissue TUBEs (Covaris 520001) with attached adapters (Covaris 520017) and glass vials (Covaris 520010). To ensure tissues remained cold and brittle during pulverization, Covaris tissueTUBEs were briefly submerged (for $\approx 5 \mathrm{~s}$ ) in liquid nitrogen between successive rounds of pulverization (for $\approx 10$ to 20 seconds) using the chilled hammer on the cold metallic block. The tissueTUBE was gently tapped so all tissue matter was collected at the bottom of the bag prior to each pulverization. To ensure that the tissue was fully pulverized, we briefly examined the tissue sample within the transparent tissueTUBE. Typically, complete pulverization takes about 5 to 10 rounds of pulverization, depending on tissue makeup and histology. Once the tissue had been pulverized to a powder, the tissueTUBEs were inverted and tissue powder was collected into attached Covaris glass vials. The subsequent tissue powder was resuspended in room temperature PBS containing protease inhibitors (Roche 11836153001) and transferred to a $15 \mathrm{ml}$ conical tube. Additional room temperature PBS containing protease inhibitors was added to $15 \mathrm{ml}$ conical tubes to a final volume of $10 \mathrm{ml}$ and tissue powder was crosslinked with $1 \%$ formaldehyde for 15 minutes while rocking at room temperature. After fixation, the crosslinking reaction was halted using $0.125 \mathrm{M}$ glycine for 5 minutes while rocking at room temperature. Crosslinked tissue powder was pelleted (750 relative centrifugal force for 5 minutes at $4^{\circ} \mathrm{C}$ ) and washed three times with cold $\left(4^{\circ} \mathrm{C}\right)$ PBS containing protease inhibitors. After each wash, crosslinked tissue powder was pelleted. Following washing, the pelleted tissue powder was subsequently stored at $-80^{\circ} \mathrm{C}$. For smaller tissue samples (less than $200 \mathrm{mg}$ ), the initial preparation and pulverization should be performed as outlined above. However, the resulting tissue powder should be resuspended with PBS containing protease inhibitors directly within the tissueTUBE, without inverting, to prevent tissue loss.
The tissue solution should then be placed in an appropriately sized container and fixed with $1 \%$ formaldehyde for 15 minutes.

\section{Chromatin immunoprecipitation and next-generation sequencing}

Antibodies for RNA polymerase II (ab5408, abcam), Rxra (sc-553, Santa Cruz Biotechnology), Ctcf (sc-5916, Santa Cruz Biotechnology and 61312, Active Motif), and Cebpa (sc-166258, Santa Cruz Biotechnology) were obtained and the ChIP assay and subsequent sequencing library preparations were conducted as previously reported [23]. The Ctcf Active Motif antibody was used for small intestine and the Ctcf sc-5916 antibody was utilized for the remaining three tissues. Sonication was performed with a Sonics Vibracell at $60 \%$ amplitude on $100 \mathrm{mg}$ aliquots of fixed tissue powder using six total 30 -second durations of sonication for all tissues. All ChIP libraries were run on an Illumina HiSeq 2000 sequencer using 50 bp single-end sequencing.

\section{Data analysis}

Sequencing reads were aligned to the genome using Bowtie [40] and binding sites were identified using the modelbased analysis of ChIP-Seq (MACS) peak caller with an mfold cutoff of 15 [41]. All position weight matrices were identified using multiple expectation maximization for motif elicitation (MEME) [42]. Normalized read depths for examining Spearman rank correlations between ChIP-seq experiments were calculated by merging 100-bp binding sites centered on peak summits for all experiments and determining the number of sequencing reads mapping to the entire list of binding sites and normalizing these values by the total number of reads that were mapped for each ChIP experiment (that is, reads per million). For RNA polymerase II promoter analyses, we determined the normalized read depth at a 2 -kb region of sequence centered on the transcription start site for all expressed genes detected by RNA-seq. To calculate the fraction of binding sites shared between ChIP-seq experiments, we divided the number of sites that overlapped by the number of sites identified in the smaller ChIP-seq dataset. Evolutionary conservation of motifs was determined by identifying all Rxra canonical motif position weight matrices within Rxro-bound sites across all tissues. GERP scores were cataloged for all base positions within the position weight matrix, and binding sites harboring motifs were compared across tissues to determine tissue specificity. For binding site conservation of sites within all peaks regardless of motif presence (Additional file 2: Figure S4), the average GERP scores for each position within a 200-bp fragment of DNA centered on peak summits was used. To determine sequencing library complexity, we calculated the fraction of uniquely mapped reads from a randomly selected 
sample of 10,000,000 reads that aligned to the mouse genome. Statistical significance was determined with a one- or two-sided Student's $t$ test.

\section{Additional files}

Additional file 1: Summary of ChIP-seq data. Table summarizing ChIP-seq experimentation across all mouse tissues. The percentage of sites shared was calculated by dividing the number of shared sites by the number of sites from the biological replicate with a smaller number of identified sites

Additional file 2: Figure S1. Canonical motifs identified in brain, small intestine, and skeletal muscle tissue samples. Figure S2. GO biological process analysis for RNA polymerase II ChIP-seq. Figure S3. GO biological process analysis for retinoid $X$ receptor ChIP-seq. Figure S4. Conservation scores relative to Rxra binding site summit. Figure S5. Analysis of ChIP-seq with Mouse ENCODE transcription factor binding site data. Figure S6. Analysis of ChIP-seq with Mouse ENCODE transcription histone modification data. Figure S7. Analysis of ChIP-seq with open chromatin annotations. Figure S8. Correlation between Rnap2 promoter enrichment and RNA-seq gene expression data. Figure S9. Rxra binding site colocalization across different liver tissue inputs.

\section{Abbreviations}

Cebpa: CCAAT/enhancer-binding protein a; ChIP: Chromatin immunoprecipitation; ChIP-seq: Chromatin immunoprecipitation nextgeneration sequencing; Ctcf: CCCTC-binding factor; GERP: Genomic evolutionary rate profiling; MACS: Model-based analysis of ChIP-Seq; MEME: Multiple expectation maximization for motif elicitation; PBS: Phosphate buffered saline; Rnap2: RNA polymerase II; RPKMs: Reads per kilobase per million mapped reads; Rxra: Retinoid $X$ receptor a.

\section{Competing interests}

The authors declare that they have no competing interests.

\section{Authors' contributions \\ DS participated in designing the study, performing ChIP-seq experimentation, data analysis and in the drafting and revision of the manuscript. JG helped design the study, run ChIP-seq experimentation, and participated in data analysis and in manuscript revision. PJ helped in data analysis and in manuscript revision. GMC aided in data analysis and in revising the manuscript. RMM participated in the coordination, design, and conception of the experimentation, along with manuscript revision. All authors read and approved the final manuscript.}

\section{Acknowledgements}

This work was supported by NIH grant 1U54HG006998-0 (to RMM) and 1K99HG006922 (to JG). We would like to thank Barbara Wold, Ross Hardison, Greg Barsh, and Timothy Reddy for helpful suggestions and advice. We would also like to thank Florencia Pauli Behn, Kimberly Newberry, Emma Dean, and other members of the Myers lab for discussions and technical support.

\section{Received: 23 July 2013 Accepted: 13 August 2013}

Published: 16 September 2013

\section{References}

1. Birney E, Stamatoyannopoulos JA, Dutta A, Guigó R, Gingeras TR, Margulies EH, Weng Z, Snyder M, Dermitzakis ET, Thurman RE, Kuehn MS, Taylor CM, Neph S, Koch CM, Asthana S, Malhotra A, Adzhubei I, Greenbaum JA, Andrews RM, Flicek P, Boyle PJ, Cao H, Carter NP, Clelland GK, Davis S, Day $\mathrm{N}$, Dhami P, Dillon SC, Dorschner MO, Fiegler $\mathrm{H}$, et al: Identification and analysis of functional elements in $1 \%$ of the human genome by the ENCODE pilot project. Nature 2007, 447(7146):799-816

2. Dunham I, Kundaje A, Aldred SF, Collins PJ, Davis CA, Doyle F, Epstein CB, Frietze S, Harrow J, Kaul R, Khatun J, Lajoie BR, Landt SG, Lee B-K, Pauli F, Rosenbloom KR, Sabo P, Safi A, Sanyal A, Shoresh N, Simon JM, Song L, Trinklein ND, Altshuler RC, Birney E, Brown JB, Cheng C, Djebali S, Dong X,
Ernst J, et al: An integrated encyclopedia of DNA elements in the human genome. Nature 2012, 489(7414):57-74.

3. Varley KE, Gertz J, Bowling KM, Parker SL, Reddy TE, Pauli-Behn F, Cross MK Williams BA, Stamatoyannopoulos JA, Crawford GE, Absher DM, Wold BJ, Myers RM: Dynamic DNA methylation across diverse human cell lines and tissues. Genome Res 2013, 23(3):555-567.

4. Ernst J, Kheradpour P, Mikkelsen TS, Shoresh N, Ward LD, Epstein CB, Zhang X, Wang L, Issner R, Coyne M, Ku M, Durham T, Kellis M, Bernstein BE: Mapping and analysis of chromatin state dynamics in nine human cell types. Nature 2011, 473(7345):43-49.

5. Lieberman-Aiden E, van Berkum NL, Williams L, Imakaev M, Ragoczy T, Telling A, Amit I, Lajoie BR, Sabo PJ, Dorschner MO, Sandstrom R, Bernstein B, Bender MA, Groudine M, Gnirke A, Stamatoyannopoulos J, Mirny LA, Lander ES, Dekker J: Comprehensive mapping of long-range interactions reveals folding principles of the human genome. Science 2009, 326(5950):289-293.

6. Sanyal A, Lajoie BR, Jain G, Dekker J: The long-range interaction landscape of gene promoters. Nature 2012, 489(7414):109-113.

7. Johnson DS, Mortazavi A, Myers RM, Wold B: Genome-wide mapping of in vivo protein-DNA interactions. Science 2007, 316(5830):1497-1502.

8. Jothi R, Cuddapah S, Barski A, Cui K, Zhao K: Genome-wide identification of in vivo protein-DNA binding sites from ChIP-Seq data. Nucleic Acids Res 2008, 36(16):5221-5231.

9. Kagey MH, Newman JJ, Bilodeau S, Zhan Y, Orlando DA, van Berkum NL, Ebmeier CC, Goossens J, Rahl PB, Levine SS, Taatjes DJ, Dekker J, Young RA: Mediator and cohesin connect gene expression and chromatin architecture. Nature 2010, 467(7314):430-435.

10. Ciofani M, Madar A, Galan C, Sellars M, Mace K, Pauli F, Agarwal A, Huang W, Parkurst CN, Muratet M, Newberry KM, Meadows S, Greenfield A, Yang Y, Jain P, Kirigin FK, Birchmeier C, Wagner EF, Murphy KM, Myers RM, Bonneau $\mathrm{R}$, Littman DR: A validated regulatory network for Th17 cell specification. Cell 2012, 151(2):289-303.

11. Kadauke S, Udugama MI, Pawlicki JM, Achtman JC, Jain DP, Cheng Y, Hardison RC, Blobel GA: Tissue-specific mitotic bookmarking by hematopoietic transcription factor GATA1. Cell 2012, 150(4):725-737.

12. Heinz S, Benner C, Spann N, Bertolino E, Lin YC, Laslo P, Cheng JX, Murre C, Singh $H$, Glass CK: Simple combinations of lineage-determining transcription factors prime cis-regulatory elements required for macrophage and B cell identities. Mol Cell 2010, 38(4):576-589.

13. Junion G, Spivakov M, Girardot C, Braun M, Gustafson EH, Birney E, Furlong EE: A transcription factor collective defines cardiac cell fate and reflects lineage history. Cell 2012, 148(3):473-486.

14. Landry JJ, Pyl PT, Rausch T, Zichner T, Tekkedil MM, Stutz AM, Jauch A, Aiyar RS, Pau G, Delhomme N, Gagneur J, Korbel JO, Huber W, Steinmetz LM: The genomic and transcriptomic landscape of a HeLa cell line. G3 (Bethesda) 2013, 3:1213-1224.

15. Visel A, Blow MJ, Li Z, Zhang T, Akiyama JA, Holt A, Plajzer-Frick I, Shoukry M, Wright C, Chen F, Afzal V, Ren B, Rubin EM, Pennacchio LA: ChIP-seq accurately predicts tissue-specific activity of enhancers. Nature 2009, 457(7231):854-858.

16. Blow MJ, McCulley DJ, Li Z, Zhang T, Akiyama JA, Holt A, Plajzer-Frick I, Shoukry M, Wright C, Chen F, Afzal V, Bristow J, Ren B, Black BL, Rubin EM, Visel A, Pennacchio LA: ChIP-Seq identification of weakly conserved heart enhancers. Nat Genet 2010, 42(9):806-810.

17. Shen Y, Yue F, McCleary DF, Ye Z, Edsall L, Kuan S, Wagner U, Dixon J, Lee L, Lobanenkov W, Ren B: A map of the cis-regulatory sequences in the mouse genome. Nature 2012, 488(7409):116-120.

18. Visel A, Taher L, Girgis H, May D, Golonzhka O, Hoch RV, McKinsey GL, Pattabiraman K, Silberberg SN, Blow MJ, Hansen DV, Nord AS, Akiyama JA, Holt A, Hosseini R, Phouanenavong S, Plajzer-Frick I, Shoukry M, Afzal V, Kaplan T, Kriegstein AR, Rubin EM, Ovcharenko I, Pennacchio LA, Rubenstein $J$ : A high-resolution enhancer atlas of the developing telencephalon. Cell 2013, 152(4):895-908.

19. May D, Blow MJ, Kaplan T, McCulley DJ, Jensen BC, Akiyama JA, Holt A, Plajzer-Frick I, Shoukry M, Wright C, Afzal V, Simpson PC, Rubin EM, Black BL, Bristow J, Pennacchio LA, Visel A: Large-scale discovery of enhancers from human heart tissue. Nat Genet 2012, 44(1):89-93.

20. Ross-Innes CS, Stark R, Teschendorff AE, Holmes KA, Ali HR, Dunning MJ, Brown GD, Gojis O, Ellis 1O, Green AR, Ali S, Chin SF, Palmieri C, Caldas C, Carroll JS: Differential oestrogen receptor binding is associated with clinical outcome in breast cancer. Nature 2012, 481(7381):389-393. 
21. Schmidt D, Wilson MD, Spyrou C, Brown GD, Hadfield J, Odom DT: ChIPseq: using high-throughput sequencing to discover protein-DNA interactions. Methods 2009, 48(3):240-248.

22. Barrera LO, Li Z, Smith AD, Arden KC, Cavenee WK, Zhang MQ, Green RD, Ren $\mathrm{B}$ : Genome-wide mapping and analysis of active promoters in mouse embryonic stem cells and adult organs. Genome Res 2008, 18(1):46-59.

23. Reddy TE, Pauli F, Sprouse RO, Neff NF, Newberry KM, Garabedian MJ, Myers RM: Genomic determination of the glucocorticoid response reveals unexpected mechanisms of gene regulation. Genome Res 2009, 19(12):2163-2171.

24. Darlington GJ, Wang N, Hanson RW: C/EBPa: a critical regulator of genes governing integrative metabolic processes. Curr Opin Genet Dev 1995, 5(5):565-570.

25. Maclsaac KD, Lo KA, Gordon W, Motola S, Mazor T, Fraenkel E: A quantitative model of transcriptional regulation reveals the influence of binding location on expression. PLoS Comput Biol 2010, 6(4):e1000773.

26. Phillips JE, Corces VG: CTCF: master weaver of the genome. Cell 2009, 137(7):1194-1211.

27. Bulger M, Groudine M: Functional and mechanistic diversity of distal transcription enhancers. Cell 2011, 144(3):327-339.

28. De Santa F, Barozzi I, Mietton F, Ghisletti S, Polletti S, Tusi BK, Muller H, Ragoussis J, Wei CL, Natoli G: A large fraction of extragenic RNA pol II transcription sites overlap enhancers. PLoS Biol 2010, 8(5):e1000384

29. Kim TH, Abdullaev ZK, Smith AD, Ching KA, Loukinov DI, Green RD, Zhang $M Q$, Lobanenkov W, Ren B: Analysis of the vertebrate insulator protein CTCF-binding sites in the human genome. Cell 2007, 128(6):1231-1245.

30. Cooper GM, Stone EA, Asimenos G, Green ED, Batzoglou S, Sidow A: Distribution and intensity of constraint in mammalian genomic sequence. Genome Res 2005, 15(7):901-913.

31. Heintzman ND, Hon GC, Hawkins RD, Kheradpour P, Stark A, Harp LF, Ye Z, Lee LK, Stuart RK, Ching CW, Ching KA, Antosiewicz-Bourget JE, Liu H, Zhang X, Green RD, Lobanenkov W, Stewart R, Thomson JA, Crawford GE, Kellis M, Ren B: Histone modifications at human enhancers reflect global cell-type-specific gene expression. Nature 2009, 459(7243):108-112.

32. Heintzman ND, Stuart RK, Hon G, Fu Y, Ching CW, Hawkins RD, Barrera LO, Van Calcar S, Qu C, Ching KA, Wang W, Weng Z, Green RD, Crawford GE, Ren $\mathrm{B}$ : Distinct and predictive chromatin signatures of transcriptional promoters and enhancers in the human genome. Nat Genet 2007, 39(3):311-318

33. Ringrose $L$, Ehret $H$, Paro R: Distinct contributions of histone $\mathrm{H} 3$ lysine 9 and 27 methylation to locus-specific stability of polycomb complexes. Mol Cell 2004, 16(4):641-653.

34. Song L, Crawford GE: DNase-seq: a high-resolution technique for mapping active gene regulatory elements across the genome from mammalian cells. Cold Spring Harb Protoc 2010, 2010(2):pdb prot5384

35. Hesselberth JR, Chen X, Zhang Z, Sabo PJ, Sandstrom R, Reynolds AP, Thurman RE, Neph S, Kuehn MS, Noble WS, Fields S, Stamatoyannopoulos JA: Global mapping of protein-DNA interactions in vivo by digital genomic footprinting. Nat Methods 2009, 6(4):283-289.

36. Mortazavi A, Williams BA, McCue K, Schaeffer L, Wold B: Mapping and quantifying mammalian transcriptomes by RNA-Seq. Nat Methods 2008, 5(7):621-628.

37. Sohlenius-Sternbeck AK: Determination of the hepatocellularity number for human, dog, rabbit, rat and mouse livers from protein concentration measurements. Toxicol In Vitro 2006, 20(8):1582-1586.

38. Yin H, Sweeney S, Raha D, Snyder M, Lin H: A high-resolution wholegenome map of key chromatin modifications in the adult Drosophila melanogaster. PLoS Genet 2011, 7(12):e1002380.

39. Lei H, Liu J, Fukushige T, Fire A, Krause M: Caudal-like PAL-1 directly activates the bodywall muscle module regulator hlh-1 in C. elegans to initiate the embryonic muscle gene regulatory network. Development 2009, 136(8):1241-1249.

40. Langmead B, Trapnell C, Pop M, Salzberg SL: Ultrafast and memory-efficient alignment of short DNA sequences to the human genome. Genome Biol 2009, 10(3):R25.
41. Zhang Y, Liu T, Meyer CA, Eeckhoute J, Johnson DS, Bernstein BE, Nusbaum C, Myers RM, Brown M, Li W, Liu XS: Model-based analysis of ChIP-Seq (MACS). Genome Biol 2008, 9(9):R137.

42. Bailey TL, Williams N, Misleh C, Li WW: MEME: discovering and analyzing DNA and protein sequence motifs. Nucleic Acids Res 2006 34(Web Server issue):W369-W373.

doi:10.1186/1756-8935-6-30

Cite this article as: Savic et al:: Mapping genome-wide transcription factor binding sites in frozen tissues. Epigenetics \& Chromatin 2013 6:30.

\section{Submit your next manuscript to BioMed Central and take full advantage of:}

- Convenient online submission

- Thorough peer review

- No space constraints or color figure charges

- Immediate publication on acceptance

- Inclusion in PubMed, CAS, Scopus and Google Scholar

- Research which is freely available for redistribution

Submit your manuscript at www.biomedcentral.com/submit
C) Biomed Central 